\title{
HENRI GAUDIER
}

\section{Algèbres de groupe du groupe additif}

Bulletin de la S. M. F., tome 117, no 2 (1989), p. 233-245

<http://www.numdam.org/item?id=BSMF_1989_117_2_233_0>

(C) Bulletin de la S. M. F., 1989, tous droits réservés.

L'accès aux archives de la revue «Bulletin de la S. M. F. » (http: //smf.emath.fr/Publications/Bulletin/Presentation.html) implique l'accord avec les conditions générales d'utilisation (http:/www.numdam.org/ conditions). Toute utilisation commerciale ou impression systématique est constitutive d'une infraction pénale. Toute copie ou impression de ce fichier doit contenir la présente mention de copyright.

\section{Numdam}




\title{
ALGĖBRES DE GROUPE DU GROUPE ADDITIF
}

\author{
PAR \\ HENRI GAUDIER $(*)$
}

\begin{abstract}
RÉSUMÉ. - Soit $k$ un corps parfait de caractéristique positive, soient $\alpha$ le groupe additif et $W$ l'anneau des vecteurs de Witt. Nous calculons l'algèbre de groupe et la $W$-algèbre de groupe de $\alpha$ au sens des $k$-schémas affines. Le résultat obtenu est ensuite étendu au cas sans caractéristique : nous obtenons alors une algèbre de puissances fractionnaires divisées sur l'anneau des gros vecteurs de Witt.
\end{abstract}

ABSTRACT. - Let $k$ be a perfect field of positive characteristic, let $\alpha$ be the additive group and let $W$ be the Witt vector ring. We compute the group algebra and the group $W$-algebra of $\alpha$ in the sense of affine schemes. The calculation is next extended to the caracteristic-free case. We so obtain a $\mathbf{W}$-algebra with fractional divided powers, where $\mathbf{W}$ denote the ring of the big Witt vectors.

Soit $\mathbf{k}$ un corps parfait de caractéristique $p$ non nulle, $\mathbf{W}$ le schéma en anneaux des gros vecteurs de Witt, et $W$ le schéma en anneaux des $p$-vecteurs de Witt. On sait ([G2]) que le foncteur oubli qui à un $\mathbf{k}$-schéma en anneaux $A$ associe son monoïde multiplicatif $A^{\times}$, admet un adjoint à gauche qui à un k-monoïde $X$ associe son algèbre de monoïde $L X$. De plus la catégorie des représentations de $X$ dans un groupe unipotent commutatif est équivalente à la catégorie des $L X$-modules.

Le but de cet article est de déterminer explicitement l'algèbre de groupe $L \alpha$ du groupe additif $\alpha$. On obtiendra ainsi une équivalence entre représentations de $\alpha$ et $L \alpha$-modules. On détermine également la structure de l'anneau $W \alpha=L \alpha \otimes W$ (où le produit tensoriel est pris au sens des schémas en anneaux [G1]), ce qui donne une équivalence entre représentations $W$-linéaires de $\alpha$ et $W \alpha$-modules. L'anneau $W \alpha$ obtenu peut être considéré comme l'algèbre des puissances divisées en une variable sur $W$ avec des puissances fractionnaires à dénominateurs les puissances de $p$.

(*) Texte reçu le 10 décembre 1987 , révisé le 15 mars 1988.

Henri Gaudier, Université Louis Pasteur, IRMA, 7 rue René Descartes, 67084 Strasbourg Cedex, France.

Adresse actuelle : Département de mathématiques, Université de Valenciennes et du Hainaut-Cambrésis, Le Mont Houy, 59326 Valenciennes Cedex, France. 
Dans la première partie de l'article on définit les coefficients *-binômiaux : ce sont des éléments de $\mathbf{W}(\mathbb{Z})$ qui ont un comportement analogue à celui des coefficients binômiaux et qui interviennent comme constantes de la multiplication dans $L \alpha$ et $W \alpha$. Dans les parties 2 et 3 , on détermine les multiplications respectives de $L \alpha$ et $W \alpha$. Dans la quatrième partie on construit, hors caractéristique, un anneau $\mathbf{W} \alpha$ qui étend l'anneau $W \alpha$ et qui peut être considéré comme la $\mathbf{W}$-algèbre des puissances divisées en une variable avec des puissances fractionnaires quelconques.

Notations. - Soit k l'anneau de base, qu'on commencera par supposer quelconque. On considère le triangle commutatif de $\mathbf{k}$-foncteurs en anneaux ([D.G. p. 639], [H p. 121]) :

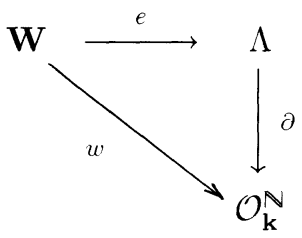

où $\mathbf{W}$ est l'anneau des gros vecteurs de Witt, $\Lambda$ est le $\lambda$-anneau universel, $\mathcal{O}_{\mathbf{k}}$ est le foncteur identité, $e$ est l'isomorphisme d'anneaux défini par :

$$
e\left(x_{1}, \ldots, x_{n}, \ldots\right)=\prod_{i \geq 1}\left(1-x_{i} T^{i}\right)^{-1}
$$

où $w$ est donné par :

$$
w_{n}\left(x_{1}, \ldots, x_{n}, \ldots\right)=\sum_{d \mid n} d x_{d}^{n / d}
$$

et où $\partial_{n}(S)$ est le coefficient de $T^{n-1}$ dans la série $\frac{d \log S}{d T}$. On sait que $w$ et $\partial$ sont des isomorphismes si k est une $\mathbb{Q}$-algèbre, par conséquent $\mathbf{W}(R)$ est isomorphe à $R^{\mathbb{N}}$ pour toute $\mathbb{Q}$-algèbre $R$.

On notera $V_{l}$ (resp. $F_{l}$ ) les morphismes de décalage (resp. de Frobenius) de $\mathbf{W}$ ou $\Lambda$, et $\sigma$ le morphisme de Teichmuller $\mathcal{O}_{\mathbf{k}} \rightarrow \mathbf{W}$ défini par $\sigma(x)=(x, 0, \ldots, 0, \ldots)$, on sait que $\sigma(x y)=\sigma(x) \sigma(y)$. Enfin si $i$ et $j$ sont des entiers on notera $((i, j))$ le coefficient binômial $\left(\begin{array}{c}i+j \\ i\end{array}\right)$. 


\section{Coefficients $*$-binômiaux et $p$-binômiaux}

1.1. Défnition. - Soient $i$ et $j$ deux entiers naturels, on appelle coefficient *-binômial * $((i, j))$ l'unique élément de $\mathbf{W}(\mathbb{Q})$ tel qu'on ait pour tout $n: w_{n}\left({ }^{*}((i, j))\right)=((n i, n j))$. Si $l$ est un entier non nul on posera également $*((i, j ; l))={ }^{*}((i, j)) / l$.

Dans la première partie de ce travail nous allons étudier ces coefficients *-binômiaux et, en particulier, montrer qu'ils sont à composantes entières.

1.2. Proposition. - Soit $k \in \mathbb{N}^{*}$, on a les identités suivantes :

(a) dans $\mathbf{W}(\mathbb{Q}[X, Y])$

$$
\sigma\left((X+Y)^{k}\right)=\sum_{l \geq 1} \sum_{\substack{i+j=l k \\(i, j, l)=1}} V_{l}\left({ }^{*}((i, j ; l)) \sigma\left(X^{i} Y^{j}\right)\right)
$$

(b) dans $\Lambda(\mathbb{Q}[X, Y])$

$$
\left(1-(X+Y)^{k} T\right)^{-1}=\prod_{\substack{r \geq 1 \\ l \geq 1}} \prod_{\substack{i+j=l k \\(i, j, l)=1}}\left(1-{ }^{*}((i, j ; l))_{r} X^{r i} Y^{r j} T^{r l}\right)^{-1}
$$

où les $*((i, j ; l))_{r}$ sont les composantes de* $((i, j ; l))$.

Puisque l'on est en caractéristique 0 , il suffit de transformer les deux membres par $w$. Or si on désigne par $D$ le membre de droite de (1.2.1), on a :

$$
\begin{aligned}
w_{n}(D) & =\sum_{l \in \mathbb{N}^{*}} \sum_{i, j} w_{n} V_{l}\left(l^{-1 *}((i, j)) \sigma\left(X^{i} Y^{j}\right)\right), \\
& =\sum_{l \mid n} \sum_{i, j} w_{n / l}\left({ }^{*}((i, j)) \sigma\left(X^{i} Y^{j}\right)\right),
\end{aligned}
$$

puisque $w_{n} V_{l}$ est égal à $l w_{n / l}$, si $l$ divise $n$, et est nul sinon,

$$
\begin{aligned}
& =\sum_{l \mid n} \sum_{i, j} w_{n / l}\left({ }^{*}((i, j))\right) w_{n / l}\left(\sigma\left(X^{i} Y^{j}\right)\right) \\
& =\sum_{l \mid n} \sum_{i, j}((n i / l, n j / l)) X^{n i / l} Y^{n j / l} \\
& =\sum_{l d=n} \sum_{\substack{i+j=l k \\
(i, j, l)=1}}((d i, d j)) X^{d i} Y^{d j} \\
& =\sum_{d \mid n} \sum_{\substack{d i+d j=n k \\
(d i, d j, n)=d}}((d i, d j)) X^{d i} Y^{d j} \\
& =(X+Y)^{n k}=w_{n}\left(\sigma(X+Y)^{k}\right)
\end{aligned}
$$


Ce qui démontre la relation (1.2.1). La relation (1.2.2) n'en est que la traduction par l'isomorphisme $e$.

1.3. Corollaire. - Si $l$ divise $i+j$ et si $l$, $i$ et $j$ sont premiers entre eux, le coefficient ${ }^{*}((i, j ; l))$ appartient $\grave{a} \mathbf{W}(\mathbb{Z})$, et la relation $(1.2 .1)$ (resp. (1.2.2)) est vraie dans $\mathbf{W}(\mathbb{Z}[X, Y])$ (resp. $\Lambda(\mathbb{Z}[X, Y])$ ).

Il est facile de voir que pour tout entier $n$ on a une décomposition

$$
1-(X+Y)^{k} T=\prod_{i=1}^{n} \prod_{j}\left(1-M_{i, j} T^{i}\right) \quad\left(\bmod T^{n+1}\right),
$$

où les $M_{i, j}$ sont des monômes de degré total $k i$. Si l'on impose de plus que les monômes aient des degrés en $X$ et $Y$ distincts, alors une telle décomposition est unique dans $\mathbb{Q}$ comme dans $\mathbb{Z}$. Passant à la limite sur $n$, on constate alors que cette décomposition est donnée par la formule (1.2.2), et que les coefficients de cette dernière sont donc dans $\mathbb{Z}$.

1.4. Coefficients *-multinômiaux. - Il est clair que la définition (1.1) peut s'étendre aux coefficients multinômiaux : si $i_{1}, \ldots, i_{h}$ sont des entiers et $l$ un entier non nul, alors le coefficient $*$-multinômial * $\left(\left(i_{1}, \ldots, i_{h} ; l\right)\right)$ est défini par :

$$
w_{n}\left({ }^{*}\left(\left(i_{1}, \ldots, i_{h} ; l\right)\right)\right)=l^{-1}\left(\left(n i_{1}, \ldots, n i_{h}\right)\right) .
$$

Comme pour les coefficients binômiaux on a les formules :

$$
\sigma\left(X_{1}+\cdots+X_{h}\right)^{k}=\sum_{\substack{i_{1}+\cdots+i_{h}=l k \\\left(i_{1}, \ldots, i_{h}, l\right)=1}} V_{l}\left({ }^{*}\left(\left(i_{1}, \ldots, i_{h} ; l\right)\right) \sigma\left(X_{1}^{i_{1}} \ldots X_{h}^{i_{h}}\right)\right)
$$

dans $\mathbf{W}\left(\mathbb{Q}\left[X_{1}, \ldots, X_{h}\right]\right)$, et

$$
\begin{aligned}
& \left(1-\left(X_{1}+\cdots+X_{h}\right)^{k} T\right)^{-1} \\
& =\prod_{\substack{r \geq 1 \\
l \geq 1}} \prod_{\substack{i_{1}+\cdots+i_{h}=l k \\
\left(i_{1}, \ldots, i_{h}, l\right)=1}}\left(1-{ }^{*}\left(\left(i_{1}, \ldots, i_{h} ; l\right)\right)_{r} X_{1}^{r i_{1}} \ldots X_{h}^{r i_{h}} T^{r l}\right)^{-1} \text {. }
\end{aligned}
$$

dans $\Lambda\left(\mathbb{Q}\left[X_{1}, \ldots, X_{h}\right]\right)$.

Et par le même raisonnement on conclut que les coefficients $*$-multinômiaux sont à composantes entières si $\left(i_{1}, \ldots, i_{k}, l\right)=1$ et si $l$ divise $\sum_{\alpha=1}^{h} i_{\alpha}$.

On appellera enfin $*$-factorielle, et on désignera par ${ }^{*} h$ ! le coefficient *-multinômial dans lequel $i_{1}=\ldots=i_{h}=l=1$.

TOME $117-1989-\mathrm{N}^{\mathrm{O}} 2$ 
1.5. Proposition. - Les coefficients *-multinômiaux vérifient les relations suivantes:

$$
\begin{aligned}
l^{*}\left(\left(i_{1}, \ldots, i_{h} ; l\right)\right) & ={ }^{*}\left(\left(i_{1}, \ldots, i_{h}\right)\right), \\
F_{k}{ }^{*}\left(\left(i_{1}, \ldots, i_{h} ; l\right)\right) & ={ }^{*}\left(\left(k i_{1}, \ldots, k i_{h} ; l\right)\right), \\
{ }^{*}\left(\left(i_{1}, \ldots, i_{h} ; l\right)\right) & =\frac{1}{l} \frac{{ }^{*}\left(i_{1}+\cdots+i_{h}\right) !}{{ }^{*} i_{1} ! \ldots{ }^{*} i_{h} !}, \\
F_{k}\left({ }^{*} h !\right) & =\frac{{ }^{*} h k !}{\left({ }^{*} k !\right)^{h}}
\end{aligned}
$$

où $F_{k}$ est le morphisme de Frobenius.

La relation (1.5.1) découle immédiatement de la définition. Pour la relation (1.5.2) on a :

$$
\begin{aligned}
w_{m} \circ F_{k}\left({ }^{*}\left(\left(i_{1}, \ldots, i_{h} ; l\right)\right)\right) & =w_{m k}\left({ }^{*}\left(\left(i_{1}, \ldots, i_{h} ; l\right)\right)\right) \\
=l^{-1}\left(\left(m k i_{1}, \ldots, m k i_{h}\right)\right) & =w_{m}\left({ }^{*}\left(\left(k i_{1}, \ldots, k i_{h} ; l\right)\right)\right) .
\end{aligned}
$$

Pour la relation (1.5.3), puisque :

$$
w_{m}\left({ }^{*} i\right)=\frac{(m i) !}{m !^{i}}
$$

on a :

$$
\begin{aligned}
w_{m}\left(\frac{*\left(i_{1}+\cdots+i_{h}\right) !}{{ }^{*} i_{1} ! \ldots{ }^{*} i_{h} !}\right) & =\frac{\left(m i_{1}+\cdots+m i_{h}\right) !}{m !^{i_{1}+\cdots+i_{h}}} \times \frac{m !^{i_{1}} \ldots m !^{i_{h}}}{\left(m i_{1}\right) ! \ldots\left(m i_{h}\right) !} \\
& =w_{m}\left({ }^{*}\left(\left(i_{1}, \ldots, i_{h}\right)\right)\right) .
\end{aligned}
$$

La relation (1.5.4) se déduit des précédentes.

1.6. Corollaire. - Pour tout $n \in N^{*}$ on $a$ :

$$
{ }^{*} n !=\prod_{i=1}^{n} *((i-1,1)),
$$

et ${ }^{*} n$ ! est divisible par $n !$.

La première relation est une conséquence immédiate de (1.5.3). Et puisque $^{*}((i-1,1))=i^{*}((i-1,1 ; i))$ on a :

$$
{ }^{*} n !=n ! \prod_{i=1}^{n}{ }^{*}((i-1,1 ; i)) .
$$

On remarquera que la première composante de ${ }^{*} n ! / n !$ est égale à 1 . 
1.7. Coefficients $p$-binômiaux et $p$-multinômiaux. - Soit maintenant $p$ un nombre premier, $W$ l'anneau des $p$-vecteurs de Witt et $V$ (resp. $F$ ) le décalage (resp. le Frobenius) dans $W$. Le morphisme $\pi: \mathbf{W} \rightarrow W$ tel que :

$$
\pi\left(w_{1}, \ldots, w_{n}, \ldots\right)=\left(w_{1}, w_{p}, \ldots, w_{p^{n}}, \ldots\right)
$$

est un homomorphisme d'anneaux. Il transforme donc la formule (1.2.1) en :

$$
\pi \circ \sigma(X+Y)^{k}=\sum^{\prime} \pi \circ V_{l}\left({ }^{*}((i, j ; l)) \sigma\left(X^{i} Y^{j}\right)\right) .
$$

Posant alors $\tau=\pi \circ \sigma$ on obtient, puisque $\pi \circ V_{l}=V^{s} \circ \pi$ si $l=p^{s}$ et est nul sinon,

$$
\tau(X+Y)^{k}=\sum^{+} V^{s}\left(\pi\left[*\left(\left(i, j ; p^{s}\right)\right)\right] \tau\left(X^{i} Y^{j}\right)\right)
$$

où la somme $\sum^{+}$est étendue à tous les indices $i, j$ et $s$ tels que $i+j=p^{s} k$ et $s=0$ ou $(i, j, p)=1$. On en déduit alors :

COROllaire. - Appelons coefficients p-binômiaux les p-vecteurs de Witt $^{p}((i, j ; s))=\pi\left[{ }^{*}\left(\left(i, j ; p^{s}\right)\right)\right]$, et soit $k$ un entier premier à $p$. Dans $W(\mathbb{Z}[X, Y])$, on a la relation :

$$
\tau(X+Y)^{k}=\sum^{\prime \prime} V^{s}\left[{ }^{p}((i, j ; s)) \tau\left(X^{i} Y^{j}\right)\right]
$$

ò̀ la somme $\sum$ est étendue à tous les indices $i, j$ et $s$ tels que $i+j=p^{s} k$ et $(i, j, p)=1$.

Si l'on définit les $p$-factorielles par ${ }^{p} i !=\pi\left({ }^{*} i !\right)$, on vérifie sans difficulté que les résultats de (1.5) et (1.6) restent valables quand on remplace les coefficients $*$-binômiaux par les coefficients $p$-binômiaux et les $*$-factorielles par les $p$-factorielles. Si $s=0$ on écrira également ${ }^{p}((i, j))$ au lieu de ${ }^{p}((i, j ; 0))$.

\section{L'anneau de groupe du groupe additif}

2.1. Proposition. - Il existe sur le groupe $\Lambda$ une unique opération bilinéaire notée * telle que :

$$
\frac{1}{1-X T} \star \frac{1}{1-Y T}=\frac{(1-X T)(1-Y T)}{1-(X+Y) T} .
$$

Cette opération est associative.

TOME $117-1989-\mathrm{N}^{\mathrm{O}} 2$ 
En effet le morphisme $\rho: \mathcal{O}_{\mathbf{k}} \times \mathcal{O}_{\mathbf{k}} \rightarrow \Lambda$ défini par :

$$
\rho(a, b)=\frac{(1-a T)(1-b T)}{1-(a+b) T}
$$

vaut 1 si $a$ ou $b$ est nul; il se prolonge donc de façon unique en une opération bilinéaire sur $\Lambda$ ([D.G. p. 631]).

En utilisant la formule (1.2.2) on obtient alors :

$$
\begin{aligned}
\frac{1}{1-X T} \star & \frac{1}{1-Y T} \\
& =\prod_{r>0} \prod_{\substack{i, j>0 \\
(i, j, i+j)=1}} \frac{1}{1-{ }^{*}((i, j ; i+j))_{r} X^{r i} Y^{r j} T^{r(i+j)}}
\end{aligned}
$$

2.2. - Soit maintenant $\mathbb{P}$ l'anneau local de $\mathbb{Z}$ en l'idéal $p \mathbb{Z}$. Supposons que $\mathbf{k}$ est une $\mathbb{P}$-algèbre.

Le groupe $\Lambda$ admet alors une décomposition $\Lambda=W^{\mathbb{N}_{p}}$ ([D.G. p. 641]) telle que l'image de $(1-X T)^{-1}$ est $\sum_{i \in \mathbb{N}_{p}} \tau\left(X^{i}\right) e_{i}([\mathrm{G} 2])$, où $\mathbb{N}_{p}$ désigne l'ensemble des entiers premiers à $p$, et $\left(e_{i}\right)$ la base (topologique) canonique de $W^{\aleph_{p}}$. L'opération $\star$ de $\Lambda$ se transporte en une opération notée également $\star$.

Proposition. - Soient $i$ et $j$ deux entiers dans $\mathbb{N}_{p}$, et $w$ et $w^{\prime}$ dans $W$. Dans $W^{\mathbf{N}_{p}}$ on a :

$$
w e_{i} \star w^{\prime} e_{j}=\sum_{k \in \mathbb{N}_{p}}\left(\sum^{\prime} V^{s}\left[{ }^{p}\left(\left(i p^{t}, j p^{u} ; s\right)\right) F^{t}(w) F^{u}\left(w^{\prime}\right)\right]\right) e_{k},
$$

où la somme $\sum^{\prime}$ est étendue aux entiers $s, t$ et $u$ tels que $i p^{t}+j p^{u}=k p^{s}$ et $\inf (s, t, u)=0$.

Dans la décomposition $\Lambda=\mathbf{W}^{\mathbb{N}_{p}}$, la relation (2.1.1) s'écrit :

$$
\begin{aligned}
\left(\sum_{i \in \mathbb{N}_{p}} \tau\left(X^{i}\right) e_{i}\right) \star & \left(\sum_{j \in \mathbb{N}_{p}} \tau\left(Y^{j}\right) e_{j}\right)= \\
& \sum_{k \in \mathbb{N}_{p}} \tau(X+Y)^{k} e_{k}-\sum_{i \in \mathbb{N}_{p}} \tau\left(X^{i}\right) e_{i}-\sum_{j \in \mathbb{N}_{p}} \tau\left(Y^{j}\right) e_{j}
\end{aligned}
$$


En utilisant (1.7.1), cette relation devient :

$$
\begin{aligned}
&\left(\sum_{i \in \mathbb{N}_{p}} \tau\left(X^{i}\right) e_{i}\right) \star\left(\sum_{j \in \mathbb{N}_{p}} \tau\left(Y^{j}\right) e_{j}\right)= \\
& \sum_{k \in \mathbb{N}_{p}}\left(\sum_{\substack{i^{\prime}+j^{\prime}=k p^{s} \\
\left(i^{\prime}, j^{\prime}, p\right)=1}} V^{s}\left[{ }^{p}\left(\left(i^{\prime}, j^{\prime} ; s\right)\right) \tau\left(X^{i^{\prime}}\right) \tau\left(Y^{j^{\prime}}\right)\right]\right) e_{k} \\
&-\sum_{i \in \mathbb{N}_{p}} \tau\left(X^{i}\right) e_{i}-\sum_{j \in \mathbb{N}_{p}} \tau\left(Y^{j}\right) e_{j} .
\end{aligned}
$$

On vérifie facilement que les termes à retrancher sont ceux qui correspondent à $i^{\prime}$ ou $j^{\prime}$ nul. Compte tenu de l'unicité de l'opération $\star$ montrée en (2.1), il suffit de vérifier que, si l'on utilise la formule (2.2.1) pour calculer $\left(\sum_{i \in \mathbb{N}_{p}} \tau\left(X^{i}\right) e_{i}\right) \star\left(\sum_{j \in \mathbb{N}_{p}} \tau\left(Y^{j}\right) e_{j}\right)$, on obtient le même résultat que ci-dessus. Cette vérification est simple.

2.3. - Supposons maintenant que $\mathbf{k}$ est un corps de caractéristique $p$. On sait que le groupe unipotent libre engendré par la droite affine est $\mathbf{Z}_{p} \times \Lambda$ ([G2]), avec l'immersion canonique $x \mapsto(1,1 /(1-x T))$. Par conséquent il existe sur $\mathbf{Z}_{p} \times \Lambda$ une unique structure d'anneau qui en fait l'anneau de groupe du groupe additif, c'est à dire que l'on a :

$$
\left(1, \frac{1}{1-x T}\right) \star\left(1, \frac{1}{1-y T}\right)=\left(1, \frac{1}{1-(x+y) T}\right) .
$$

L'élément unité est $e_{0}=(1,1)$, et en décomposant $(1,1 /(1-x T))$ en $e_{0}+(0,1 /(1-x T))$, on obtient :

$$
\left(0, \frac{1}{1-x T}\right) \star\left(0, \frac{1}{1-y T}\right)=\left(0, \frac{(1-x T)(1-y T)}{1-(x+y) T}\right) .
$$

On a donc démontré :

Corollaire. - L'algèbre de groupe du groupe additif est le groupe $L \alpha=\mathbf{Z}_{p} \times \prod_{i \in \mathbb{N}_{p}} W$ muni de la multiplication définie par la formule (2.2.1) et par

$$
n e_{0} \star n^{\prime} e_{0}=n n^{\prime} e_{0} \quad \text { et } n e_{0} \star w e_{i}=n w e_{i}
$$




\subsection{Remarques.}

1) Dans la Proposition (2.2) les conditions sur $s, t$ et $u$ entraînent que deux d'entre eux sont nuls. La formule (2.2.1) peut donc s'écrire :

$$
\begin{aligned}
& w e_{i} \star w^{\prime} e_{j}=\sum_{t>0} p\left(\left(i p^{t}, j\right)\right) F^{t}(w) w^{\prime} e_{i p^{t}+j} \\
& +\sum_{u>0}{ }^{p}\left(\left(i, j p^{u}\right)\right) w F^{u}\left(w^{\prime}\right) e_{i+j p^{u}}+V^{s}\left[^{p}((i, j ; s)) w w^{\prime}\right] e_{(i+j) p^{-s}}
\end{aligned}
$$

où $s$ est la valuation $p$-adique de $i+j$.

2) Désignons par $c_{i, j, k}\left(w, w^{\prime}\right)$ le coefficient de $e_{k}$ dans $w e_{i} \star w^{\prime} e_{j}$. En utilisant (1.5.1), on obtient :

$$
\begin{aligned}
F^{s}\left(c_{i, j, k}\left(w, w^{\prime}\right)\right) & ={ }^{p}\left(\left(i p^{t}, j p^{u}\right)\right) F^{t}(w) F^{u}\left(w^{\prime}\right), \text { si } k p^{s}=i p^{t}+j p^{u} \\
c_{i, j, k}\left(w, w^{\prime}\right) & =0 \quad \text { sinon. }
\end{aligned}
$$

\section{La $W$-algèbre du groupe additif}

On suppose toujours que $\mathbf{k}$ est un corps de caractéristique $p$. Si $M$ est un groupe affine commutatif, on sait qu'il est équivalent de se donner une opération du groupe additif sur $M$ ou de se donner une structure de $L \alpha$-module ([G2]). Si maintenant $M$ est un $W$-module affine, il est équivalent de se donner une opération $W$-linéaire du groupe additif ou de se donner une structure de $L \alpha$-module compatible avec la structure de $W$-module et donc d'après ([G1]) une structure de $W \otimes L \alpha$-module. Le but de cette partie est de donner une expression simple de cette $W$-algèbre.

3.1. LemME. - Le morphisme de groupe $W \otimes L \alpha \rightarrow W^{\mathrm{N}[1 / p]}$ défini par

$$
\begin{aligned}
w \otimes\left(n e_{0}+\right. & \left.\sum_{i \in \mathbb{N}_{p}} w_{i} e_{i}\right) \\
& \longmapsto n w f_{0}+\sum_{j \in \mathbb{N}[1 / p]^{*}} F^{v(j)}-(w) F^{v(j)}+\left(w_{j p^{-v(j)}}\right) f_{j}
\end{aligned}
$$

est un isomorphisme. On note $\left(f_{j}\right)$ la base canonique de $W^{\mathbb{N}[1 / p]}$, si $j \in \mathbb{N}[1 / p]^{*}, v(j)$ désigne sa valuation $p$-adique, et si $n \in \mathbb{Z}$ on pose $n_{+}=\sup (n, 0)$ et $n_{-}=\sup (-n, 0)$.

En effet on a un isomorphisme $W \otimes W \stackrel{\sim}{\longrightarrow} W^{\mathbb{Z}}$ par conséquent $W \otimes W^{\mathbb{N}_{p}} \stackrel{\sim}{\longrightarrow} W^{\mathbf{Z} \times \mathbb{N}_{p}}$. Et la bijection $\mathbb{Z} \times \mathbb{N}_{p} \stackrel{\sim}{\longrightarrow} \mathbb{N}[1 / p]^{*}$ définie par 
$(n, i) \mapsto i p^{n}$ donne un isomorphisme $W \otimes W^{\mathbb{N}_{p}} \stackrel{\sim}{\longrightarrow} W^{\mathbb{N}[1 / p]^{*}}$ dont on vérifie aisément qu'il est donné par (3.1.1).

3.2. ThÉoRÈme. - Soit $W \alpha=W^{\mathbb{N}[1 / p]}$ la $W$-algèbre de groupe $d u$ groupe additif. Si $i$ et $j$ sont dans $\mathbb{N}[1 / p]$, posons $[i]=v_{p}(i)_{-}$, $[i, j]=\sup ([i],[j])$ et $\delta(i, j)=[i, j]-[i+j]$. Alors la multiplication dans $W \alpha$ est donnée par :

$$
\begin{aligned}
& x f_{i} \star y f_{j}= \\
& V^{\delta(i, j)}\left[p\left(\left(i p^{[i, j]}, j p^{[i, j]} ; \delta(i, j)\right)\right) F^{[i, j]-[i]}(x) F^{[i, j]-[j]}(y)\right] f_{i+j} .
\end{aligned}
$$

En raison de l'unicité de l'algèbre de groupe et du lemme (3.1), on sait qu'il existe une unique multiplication sur $W \alpha$ telle que le morphisme composé

$$
\begin{aligned}
& W \quad \longrightarrow W \otimes L \alpha \longrightarrow W \alpha \\
& w \quad \longmapsto \quad w \otimes 1 \quad \longmapsto \quad w f_{0}
\end{aligned}
$$

soit un morphisme d'anneau et que le morphisme composé

$$
\begin{aligned}
& \alpha \longrightarrow W \otimes L \alpha \quad \longrightarrow \quad W \alpha \\
& x \longmapsto 1 \otimes\left(e_{0}+\sum_{i \in \mathbb{N}_{p}} \tau x^{i} e_{i}\right) \longmapsto \sum_{i \in \mathbb{N}[1 / p]} \tau x^{i p^{[i]}} f_{i}
\end{aligned}
$$

transforme l'addition de $\alpha$ en la multiplication de $W \alpha$. Il suffit donc de vérifier que la multiplication donnée par (3.2.1) vérifie ces deux propriétés. Pour la première c'est évident. Pour la seconde on doit vérifier que :

$$
\sum_{k \in \mathbb{N}[1 / p]} \tau(x+y)^{k p^{[k]}} f_{k}=\left(\sum_{i \in \mathbb{N}[1 / p]} \tau x^{i p^{[i]}} f_{i}\right) \star\left(\sum_{j \in \mathbb{N}[1 / p]} \tau y^{j p^{[j]}} f_{j}\right) .
$$

On doit donc avoir pour tout $k$ :

$$
\begin{gathered}
\tau(x+y)^{k p^{[k]}}= \\
\sum_{i+j=k} V^{\delta(i, j)}\left[{ }^{p}\left(\left(i p^{[i, j]}, j p^{[i, j]} ; \delta(i, j)\right)\right) F^{[i, j]-[i]}\left(\tau x^{i p^{[i]}}\right) F^{[i, j]-[j]}\left(\tau y^{j p^{[j]}}\right)\right] f_{k}
\end{gathered}
$$

Calculons alors la composante fantôme $\Phi_{m}$ :

$$
\begin{aligned}
(x+y)^{k p^{[k]+m}} & =\sum_{i+j=k} \Phi_{m} V^{\delta(i, j)}\left[{ }^{p}\left(\left(i p^{[i, j]}, j p^{[i, j]} ; \delta(i, j)\right)\right) \tau x^{i p^{[i, j]}} \tau y^{\left.j p^{[i, j]}\right]}\right] \\
& =\sum_{\substack{i+j=k \\
\delta(i, j) \leq m}} \Phi_{m-\delta(i, j)}\left[{ }^{p}\left(\left(i p^{[i, j]}, j p^{[i, j]}\right)\right) \tau x^{i p^{[i, j]}} \tau y^{\left.j p^{[i, j]}\right]}\right] \\
& =\sum_{\substack{i+j=k \\
\delta(i, j) \leq m}}\left(\left(i p^{m+[k]}, j p^{m+[k]}\right)\right) x^{i p^{m+[k]}} y^{j p^{m+[k]}} \cdot
\end{aligned}
$$

TOME $117-1989-\mathrm{N}^{\mathrm{o}} 2$ 
Il suffit alors de voir que l'on a simplement la formule du binôme pour achever la démonstration.

3.3. Remarque. - Il résulte du théorème que l'on a quelques soient $i$ et $j$ dans $\mathbb{N}[1 / p]$ :

$$
\begin{aligned}
& F^{[i]}(x) f_{i} \star F^{[j]}(y) f_{j}= \\
& V^{\delta(i, j)}\left[{ }^{p}\left(\left(i p^{[i, j]}, j p^{[i, j]} ; \delta(i, j)\right)\right)\right] F^{[i+j]}(x y) f_{i+j} .
\end{aligned}
$$

3.4. Corollaire. - La sous-algèbre $\bigoplus_{i \in \mathbb{N}} W f_{i}$ de Wo est isomorphe à l'algèbre des puissances divisées en une variable sur $W$.

En effet si $i$ et $j$ sont entiers, on a en utilisant (3.2.1) et (1.5.3) :

$$
\left({ }^{p} i ! f_{i}\right) \star\left({ }^{p} j ! f_{j}\right)={ }^{p}((i, j)){ }^{p} i !{ }^{p} j ! f_{i+j}={ }^{p}(i+j) ! f_{i+j} .
$$

Par conséquent si l'on pose $X=1 . f_{1}$, on obtient ${ }^{p} i ! f_{i}=X^{\star i}$. La sousalgèbre $\bigoplus_{i \in \mathbb{N}}$ est donc analogue à l'algèbre des puissances divisées. Mais les factorielles et les coefficients binômiaux ont été remplacés par les $p$-factorielles et les coefficients $p$-binômiaux. Mais on a remarqué (1.6) que la première composante de ${ }^{*} n ! / n$ ! est égale à 1 , il en est de même pour ${ }^{p} n ! / n$ ! qui est donc inversible, puisque l'on est en caractéristique $p$.

\section{Algèbre des puissances fractionnaires divisées}

Dans ce paragraphe $\mathbf{k}$ est un anneau commutatif quelconque. La construction de $W \alpha$ peut s'étendre à l'anneau $\mathbf{W}$ des gros vecteurs de Witt.

Soit $\mathbb{Q}_{+}$l'ensemble des rationnels positifs ou nuls. Si $i$ et $j$ sont dans $\mathbb{Q}_{+}$, on note $d(i)$ le dénominateur de $i, d(i, j)$ le p.p.c.m. de $d(i)$ et $d(j)$, et $\delta(i, j)$ le quotient $d(i, j) / d(i+j)$.

4.1. ThÉORÈme. - Soit $\mathbf{W} \alpha$ le groupe $\prod_{\mathfrak{Q}_{+}} \mathbf{W}$ et $\left(f_{i}\right)$ sa base canonique :

1) la multiplication

$$
\begin{aligned}
& \quad x f_{i} \star y f_{j}= \\
& V_{\delta(i, j)}\left[{ }^{*}((i d(i, j), j d(i, j) ; \delta(i, j))) F_{d(i, j) / d(i)}(x) F_{d(i, j) / d(j)}(y)\right] f_{i+j}
\end{aligned}
$$

fait de $\mathbf{W} \alpha$ un anneau commutatif;

2) le morphisme

$$
x \longmapsto \sum_{i \in \mathbb{Q}_{+}} \sigma\left(x^{i d(i)}\right) f_{i}
$$


est un homomorphisme du groupe additif $\alpha$ dans le groupe multiplicatif de $\mathbf{W} \alpha$.

La vérification est facile.

\subsection{Remarques.}

1) Comme dans le cas des $p$-vecteurs de Witt ( $c f .3 .3$ ) la formule (4.1.1) entraîne :

$$
F_{d(i)}(x) f_{i} \star F_{d(j)}(y) f_{j}=V_{\delta(i, j)}[*((i d(i, j), j d(i, j) ; \delta(i, j)))] F_{d(i+j)}(x y) f_{i+j}
$$

2) Il en résulte immédiatement que si $i$ et $j$ sont entiers on a :

$$
x^{*} i ! f_{i} \star y^{*} j ! f_{j}=x y^{*}(i+j) ! f_{i+j} .
$$

Ce qui montre que le sous-anneau $\bigoplus_{i \in \mathbb{N}} \mathbf{W} f_{i}$ de $\mathbf{W} \alpha$ est analogue à l'algèbre des puissances divisées. De façon plus précise, on peut vérifier que l'idéal engendré par les $\left(f_{i}\right)_{i \in \mathbb{N}^{*}}$ muni des applications $\gamma_{n}$ définies par :

$$
\gamma_{n}\left(x f_{i}\right)=F_{i}\left({ }^{*} n ! / n !\right) x^{n} f_{n i},
$$

est un idéal à puissances divisées au sens de [B].

4.3. - On peut également interpréter $\mathbf{W} \alpha$ tout entier en termes de puissances fractionnaires divisées. Soient $i$ et $j \in \mathbb{Q}_{+}$, posons

$$
{ }^{*}((i, j))=\frac{1}{d(i, j)} V_{d(i, j)}{ }^{*}((i d(i, j), j d(i, j))) .
$$

Alors * $((i, j))$ est dans $\mathbf{W}(\mathbb{Q})$, et il est facile de voir que la formule (1.5.2) reste valable. Supposons alors que $\mathbf{k}$ soit de caractéristique 0 et soit $\overline{\mathbf{W}} \alpha$ le $\mathbf{W}$-module $\mathbf{W}^{\mathbb{Q}_{+}}$, de base $\bar{f}_{i}$, muni de la multiplication :

$$
x \bar{f}_{i} \star y \bar{f}_{j}={ }^{*}((i, j)) x y \bar{f}_{i+j} .
$$

Proposition. - $\overline{\mathbf{W}} \alpha$ est une $\mathbf{W}$-pseudo-algèbre (i.e. une algèbre sans unité) et le morphisme :

$$
\begin{aligned}
& \mathbf{W} \alpha \longrightarrow \overline{\mathbf{W}} \alpha \\
& x f_{i} \longmapsto \frac{1}{d(i)} V_{d(i)}(x) \bar{f}_{i}
\end{aligned}
$$

est un homomorphisme injectif d'algèbres.

$$
\text { TOME } 117-1989-\mathrm{N}^{\mathrm{O}} 2
$$


La vérification est aisée. On trouvera dans [G3] d'autres résultats concernant les algèbres $\mathbf{W} \alpha, \overline{\mathbf{W}} \alpha$ et les coefficients $*$-binômiaux.

\section{BIBLIOGRAPHIE}

[B] Berthelot (Pierre). - Cohomologie cristalline des schémas de caractéristique $p>0$. - Berlin, Springer-Verlag, 1974 (Lecture notes in Math., 407).

[D.G.] Demazure (Michel) et Gabriel (Pierre). - Groupes algébriques. - Paris, Masson et Amsterdam, North-Holland , 1970.

[G1] Gaudier (Henri). - Sur le produit tensoriel des groupes affines, Manuscr. Math., t. 17, 1975, p. 21-54.

[G2] Gaudier (Henri). - Groupes libres et algèbres de groupes en Géométrie algébrique, Manuscr. Math., t. 25, 1978, p. 79-86.

[G3] Gaudier (Henri). - Relèvement des coefficients binômiaux dans les vecteurs de Witt. - (Actes $18^{\text {ième }}$ session Séminaire Lotharingien de Combinatoire, 1987), Publ. Math. IRMA Strasbourg, nº 358/5-18, 1988, p. 93-108.

[H] HazEwinkel (Michiel). - Formal groups and applications. - New-York, Academic Press, 1978 . 